\title{
CITOGENÉTICA DE ALGUMAS ESPÉCIES FRUTÍFERAS NATIVAS DO NORDESTE DO BRASIL ${ }^{1}$
}

\author{
ERLLENS ÉDER-SILVA², LEONARDO PESSOA FELIX ${ }^{3}$, RISELANE DE LUCENA ALCÂNTARA BRUNO $^{3}$
}

RESUMO - O presente trabalho teve como objetivo caracterizar o cariótipo de seis espécies de frutíferas nativas do Brasil por meio da análise do número e morfologia cromossômica das mesmas. As pontas de raízes jovens foram pré-tratadas com 8-hidroxiquinolina 0,002M, esmagadas em ácido acético $45 \%$ e coradas convencionalmente com Giemsa. O material estudado apresentou cariótipo simétrico, com cromossomos pequenos, medindo de 0,6 a 2,5 $\mu \mathrm{m}$, predominantemente metacêntricos a submetacêntricos com uma a duas constrições secundárias visíveis em todas as espécies, exceto em Bromelia karatas, onde não foi possível identificar a morfologia cromossômica ou a presença de constrições secundárias. Na família Myrtaceae, observou-se um complemento cromossômico diplóide com 2n=98 em Psidium arboreum Vell. e 2n=44 em P. araça Raddi; na família Bromeliaceae, 2n=50 em Bromelia karatas L.; na família Malvaceae, 2n=16 para Guazuma ulmifolia Lam.; na família Sapindaceae, 2n=32 em Talisia esculenta Radlk., e, na família Caricaceae, 2n=18 em Jaracatia spinosa (Aubl.) A. DC. Todos os dados cromossômicos apresentados neste trabalho são inéditos, exceto para a espécie T. esculenta, que teve seu registro prévio confirmado. As espécies possuem potencial para utilização no melhoramento de plantas, e uma inversão em heterozigoze parece estar envolvida na evolução cariotípica de Guazuma ulmifolia.

Termos de indexação: Evolução cariotípica, inversão cromossômica, condensação tardia.

\section{CYTOGENETICS OF SOME SPECIES OF NATIVE FRUITS FROM NORTHEAST BRAZIL}

\begin{abstract}
This work aimed to characterize the karyotype of six species of native fruits from Brazil through number and chromosome morphology analysis. The young root tips were pretreated with 8 -hydroxiquinolin $0.002 \mathrm{M}$, squashed in $45 \%$ acetic acid, and conventionally stained with Giemsa. All species showed symmetrical karyotype with small chromosomes and 0.6-2.5 $\mu$ m of length, mainly meta to sub-metacentric ones with one to two secondary visible constrictions in all species, except in Bromelia karatas in which it was not possible to identify the chromosome morphology or the presence of secondary constrictions. In Myrtaceae family, it had been observed a diploid chromosome supplement with 2n=98 in Psidium arboretum Vell. and $2 \mathrm{n}=44$ in $P$. araça Raddi; $2 \mathrm{n}=50$ in Bromelia karatas L.; in the Malvaceae, 2n=16 for Guazuma ulmifolia Lam.; in Sapindaceae, 2n=32 in Talisia esculenta Radlk. and, in Caricaceae, $2 \mathrm{n}=18$ in Jaracatia spinosa (Aubl.) A. DC. All chromosome data presented in this work have been karyologically unpublished, except for T. esculenta which had previous reports confirmed. The species are potentially useful in breeding systems, and a heterozygosis inversion seems to be involved in the karyotype evolution of Guazuma ulmifolia.
\end{abstract}

Index terms: Karyological evolution, chromosome invertion, late condensation.

\section{INTRODUÇÃO}

Exceto para algumas espécies nativas já consagradas pelo cultivo e consumo, como o abacaxi (Ananas comosus) e o maracujá (Passiflora edulis), a maioria das frutíferas nativas são citologicamente pouco conhecidas. Mesmo em espécies mais exploradas comercialmente, como o cajueiro (Anacardium occidentale) e o cajá (Spondias mombim), são raros os registros cariológicos mais detalhados, constando, na maioria das vezes, apenas do registro de número cromossômico. Exceção a essa regra, são os estudos com coloração convencional e bandeamento com fluorocromos (Melo et al., 2001) e hibridização in situ (Melo \& Guerra, 2003) para o gênero Passiflora. Por outro lado, frutíferas exóticas amplamente cultivadas no Brasil têm sido alvo de estudos cromossômicos mais detalhados, como, por exemplo, no gênero Citrus (Carvalho et al., 2005), extensamente estudado.

Desde o início do século XX, a citogenética vem sendo amplamente utilizada em trabalhos que envolvem caracterização taxonômica e estudos de evolução e filogenia (Stuessy, 1990). O destaque principal é a citotaxonomia clássica que se caracteriza pela observação do número e da morfologia dos cromossomos mitóticos e do seu comportamento na meiose (Stebbins, 1971). Esses parâmetros têm se tornado um dos mais importantes instrumentos para a compreensão das relações de parentesco e dos mecanismos envolvidos na evolução cariotípica, tanto em níveis hierárquicos inferiores (espécies, gêneros) quanto superiores (famílias).

Nos últimos anos, a citogenética alcançou importantes progressos relacionados ao desenvolvimento da biologia molecular, o que permitiu o aparecimento de novas e diversificadas técnicas citológicas, como a hibridização fluorescente in situ ou FISH (Pedrosa et al., 2002), a hibridização genômica in situ ou GISH (Poggio et al., 2005) e a microdissecção cromossômica (Forminaya et al., 2005). Essas técnicas vêm permitindo um detalhamento minucioso dos cariótipos, permitindo o reconhecimento de pequenas variações cromossômicas, difíceis de serem detectadas com técnicas convencionais. Contudo, o alto custo em drogas e dos

${ }^{1}$ (Trabalho 105-2006). Recebido em 24-07-2006. Aceito para publicação em 05-12-2006.

2 Aluno de Doutorado em Agronomia da UFPB/CCA/Campus II, Areia-Paraíba, 58397-000, tel: (83) 9932-1733; erllens_eder@hotmail.com;

${ }^{3}$ Professor (a) Doutor (a) Adjunto (a) da UFPB/CCA/Campus II, Areia-Paraíba, 58397-000, tel: (83) 3362-2300; lane@cca.ufpb.br ou 1pfelix@yahoo.com.br 
equipamentos de laboratório torna restrita sua aplicação a grupos com interesse econômico já comprovado. Desse modo, a variabilidade em número e a morfologia cromossômica continuam sendo amplamente utilizadas nas análises cariológicas (Guerra, 2000), especialmente em plantas pouco estudadas citologicamente, como é o caso das frutíferas nativas.

Os estudos cariológicos no Brasil, especialmente a partir dos anos noventa, passaram a abordar aspectos da evolução cariológica em diversos grupos de plantas do Brasil (Melo et al., 1997; Guerra e Nogueira, 2001). Todavia, em relação às frutíferas nativas, são raros os estudos cariológicos abordando aspectos de sua variabilidade cromossômica, características bastante úteis na taxonomia de alguns grupos. Em Genipa americana, por exemplo, a ocorrência de $2 \mathrm{n}=22$ para populações de Recife-PE, e de $2 n=20$ para populações de Campinas- SP, indicou claramente a ocorrência de duas espécies distintas (Pierozzi \& Cruz, 1998). Destacam-se os trabalhos de Melo et al. (2001) e Melo \& Guerra (2003) em Passiflora, os quais, além de contribuírem para uma descrição mais detalhada das espécies, são importantes indicadores das potencialidades de hibridização com vistas a programas de melhoramento. $\mathrm{O}$ presente trabalho consiste no estudo do cariótipo, a partir de uma análise do número e da morfologia cromossômica, em seis espécies de frutíferas nativas da região Nordeste do Brasil, objetivando contribuir para o conhecimento da variabilidade cariológica dessas plantas na região.

\section{MATERIAL E MÉTODOS}

Todo o material foi coletado na região Nordeste do Brasil, nos Estados da Paraíba e Ceará, durante o ano de 2004. Exsicatas de todas as espécies analisadas foram depositadas no Herbário Jayme Coelho de Moraes (EAN), do Centro de Ciências Agrárias da Universidade Federal da Paraíba. Foram estudadas seis espécies fruteiras nativas indicadas pelas comunidades locais, consumidas tanto in natura como em compotas. A Tabela 1 relaciona os táxons estudados, seus números cromossômicos, contagens prévias e o material- testemunho depositado no Herbário EAN.

Para as análises cromossômicas, foram adotadas as recomendações de Guerra \& Sousa (2002) para coloração convencional. Utilizou-se de pontas de raízes pré-tratadas com 8-hidroxiquinoleína (8-HQ) $0,002 \mathrm{M}$, por cerca de seis horas, a $4^{\circ} \mathrm{C}$ em geladeira, fixadas em Carnoy 3:1 (etanol absoluto/ácido acético glacial v/v), por um período de 3-24 horas, à temperatura ambiente e estocadas em freezer a $20^{\circ} \mathrm{C}$ até posterior análise. Para o preparo das lâminas, as raízes passaram por dois banhos de 5 minutos cada, em água destilada, e foram hidrolisadas em ácido clorídrico $5 \mathrm{~N}$, por 20 minutos, novamente lavadas em água destilada, e os meristemas esmagados em ácido acético $45 \%$. Em seguida, as lâminas foram congeladas em nitrogênio líquido para a remoção das lamínulas, secas ao ar, coradas convencionalmente com Giemsa 2\% (Guerra, 1983) e montadas em Entellan.

As células foram fotografadas com uma máquina digital Olympus D-54 adaptada a um microscópio Olympus BX41 e depois transferidas para o computador. Para a montagem das pranchas, as melhores células foram digitalizadas no programa Corel Photo Paint, utilizando-se apenas dos comandos de brilho e contraste. Nesse mesmo programa, foram obtidas as medidas cromossômicas, projetando-se em uma mesma imagem do computador a metáfase, juntamente com uma barra de escala com $10 \mu \mathrm{m}$, cuja medida em centímetro $(\mathrm{cm})$ serviu de base para a conversão em micrômetros $(\mu \mathrm{m})$ das medidas dos cromossomos.

TABELA 1 - Relação das espécies analisadas com as respectivas famílias, locais de coleta, número cromossômico observado e contagem prévia com respectiva fonte bibliográfica.

\begin{tabular}{|c|c|c|c|c|}
\hline Família/Espécie estudada & $\mathrm{N}^{\mathrm{os}}$ cromossômicos & $\begin{array}{l}\text { Locais de } \\
\text { coleta }\end{array}$ & $\begin{array}{c}\begin{array}{c}\text { Contagens } \\
\text { prévias }\end{array} \\
\end{array}$ & Fonte \\
\hline Bromeliaceae & & & & \\
\hline Bromelia karatas $\mathrm{L}$. & 50 & Areia-PB & -- & -- \\
\hline $\begin{array}{l}\text { Caricaceae } \\
\text { Jaracatia spinosa (Aubl.) A. DC. }\end{array}$ & 18 & Pacoti-CE & -- & -- \\
\hline Myrtaceae & & & & \\
\hline Psidium arboreum Vell. & 98 & Areia-PB & -- & -- \\
\hline P. araça Raddi & 44 & Areia-PB & -- & - \\
\hline $\begin{array}{l}\text { Sapindaceae } \\
\text { Talisia esculenta Radlk }\end{array}$ & 32 & Areia-PB & 32 & Carvalheira et al. (1991) \\
\hline Sterculiaceae & & & & \\
\hline Guazuma ulmifolia Lam. & 16 & Caucaia-CE & -- & -- \\
\hline
\end{tabular}

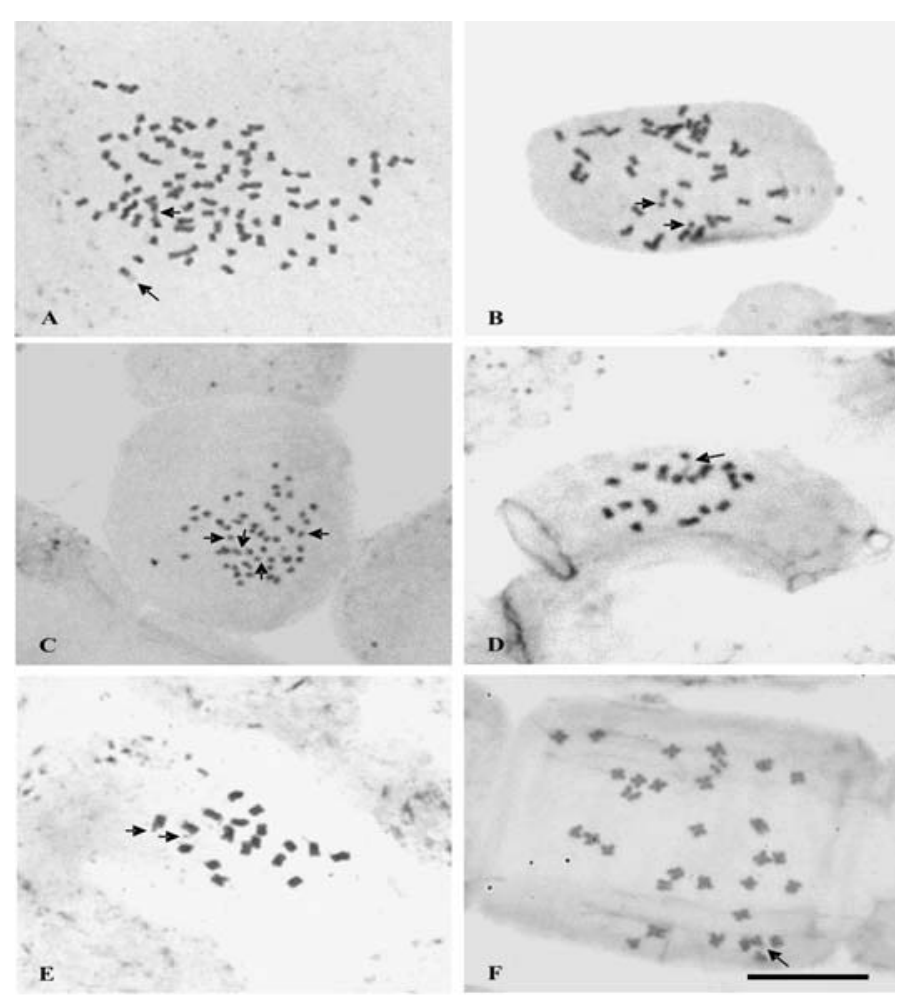

FIGURA 1 - Complemento cromossômico diplóide de frutíferas nativas do Nordeste do Brasil. A. Psidium arboreum $(2 \mathrm{n}=98) ;$ B. P. $\operatorname{araça~}(2 \mathrm{n}=44) ;$ C. Bromelia karatas $(2 \mathrm{n}=50)$; D. Guazuma ulmifolia $(2 \mathrm{n}=16) ; \mathbf{E}$. Jaracatia spinosa $(2 \mathrm{n}=18) ; \mathbf{F}$. Talisia esculenta $(2 \mathrm{n}=32)$. Setas em $\mathbf{A}, \mathbf{E}$ e $\mathbf{F}$ indicam satélites terminais; em $\mathbf{B}$ e $\mathbf{D}$, satélites em constrição secundária proximal; $\mathbf{C}$, cromossomos de condensação tardia. Barra em $\mathbf{F}=10 \mu \mathrm{m}$. 


\section{RESULTADOS E DISCUSSÃO}

As espécies analisadas apresentaram cromossomos pequenos, medindo de 0,6 a $2,5 \mu \mathrm{m}$, predominantemente metacêntricos a submetacêntricos com uma a duas constrições secundárias visíveis em todas as espécies (Figura 1A-F), exceto para Bromelia karatas (figura 1C), onde não foi possível identificar a morfologia cromossômica ou a presença de constrições secundárias. Com exceção de Talisia esculenta, as demais contagens obtidas no presente trabalho são inéditas, um fato comum em plantas tropicais, como pode ser visto nas descrições de Felix \& Guerra (1998), onde todas as contagens cromossômicas para a família Orchidaceae foram inéditas, para as 10 espécies do gênero Habenaria do Nordeste do Brasil.

Na família Myrtaceae, Psidium arboreum (araçá-boi) apresentou 2n=98 (Figura 1A), confirmando a tendência de poliploidia no gênero. O registro de $2 \mathrm{n}=44$ em Psidium acutangulum DC., uma espécie de cerrado de São Paulo (ForniMartins \& Martins, 2000), e em $P$. araça, indica uma linhagem evolutiva no gênero Psidium, com base em $\mathrm{x}=11$. $P$. arboreum, além de evolução por poliploidia, apresenta ganho aneuplóide de alguns pares cromossômicos, provavelmente resultantes de erros de disjunção meiótica. Apesar de apresentar excelentes qualidades como frutífera nativa, além de boas características nutricionais, com valores para vitamina C em torno de $286,16 \mathrm{mg} /$ $100 \mathrm{~g}$ (Éder-Silva et al., 2005a), P. arboreum é muito pouco utilizado como alimento pela população. Seu uso em programas de melhoramento parece limitado a cruzamento com espécies de Psidium que apresentem esse mesmo número básico.

Psidium araça (araçá), com 2n=44, apresentou cromossomos predominantemente metacêntricos e submetacêntricos, medindo 0,8 a $2,1 \mu \mathrm{m}$ e um par de satélites longamente distendido pela ocorrência de uma constrição secundária proximal (Figura 1B). A presente contagem coincide apenas com duas outras contagens para o gênero (Atchison, 1947; Fedorov, 1969; Forni-Martins \& Martins, 2000) e diverge de $P$. guajava, com $2 \mathrm{n}=22$ (Fedorov, 1969). O gênero é cariologicamente variável, com várias contagens com $2 \mathrm{n}=22,44$ e 88 (Fedorov, 1969; Goldblatt, 1985), sugerindo a ocorrência de uma série poliplóide no gênero e um número básico $\mathrm{x}=11$. $\mathrm{O}$ fruto dessa espécie é de grande interesse econômico, sendo amplamente utilizado na culinária (compotas, doces, cremes e patês) e no consumo in natura (Manica, 2000), comercializado em feiras livres e supermercados.

Bromelia karatas (banana de raposa) apresentou $2 \mathrm{n}=50$, divergindo das duas únicas contagens prévias para o gênero de 2n=94 em B. goeldiana (Goldblatt \& Johnson, 1994) e 2n=ca. 150 em B. laciniosa (Gitaí et al., 2005). A espécie apresentou cromossomos pequenos, medindo de 0,6 a $1,2 \mu \mathrm{m}$, com morfologia difícil de ser identificada e ocorrência de um a dois pares cromossômicos de condensação tardia (Figura 1C). Esse registro numérico é muito freqüente para a família, bem como a ocorrência de vários níveis de ploidia em outros gêneros, inclusive em Bromelia (Gitaí et al., 2005). Quanto aos cromossomos de condensação tardia, estes não têm um significado evolutivo claro, embora seja um fenômeno recorrente em outros grupos de plantas, como em orquídeas (Felix \& Guerra, 1998) ou em espécies do gênero Arachis com genoma A (Fernandez \& Krapovickas, 1994). A espécie $B$. karatas, pouco utilizada como frutífera nativa pelas populações locais, possui razoável teor de vitamina $\mathrm{C}$, apresentando potencial de uso doméstico ou na indústria alimentícia, podendo ser consumida isoladamente ou na composição de saladas (Éder-Silva et al., 2005b).

Guazuma ulmifolia (mutamba) possui cariótipo com 2n=16, ligeiramente bimodal pela ocorrência de dois pares cromossômicos maiores. Apresenta um par satelitado heteromórfico pela ocorrência de uma constrição secundária proximal, longamente distendida em um dos homólogos (Figura 1D), o que sugere uma inversão paracêntrica heterozigota. Um fenômeno parecido é registrado em Eleutherine bulbosa (Iridaceae), que apresentou, além de uma inversão, uma duplicação em tandem da RON (Guerra, 1988). O número observado para G. ulmifolia coincide com a única contagem prévia para o gênero em G. tomentosa (Goldblatt, 1981), uma espécie bastante relacionada taxonomicamente e, algumas vezes, considerada sinonímia de G. ulmifolia. Trata-se de uma planta raramente usada como frutífera nativa (Braga, 1960), sendo utilizada esporadicamente pelas populações locais da microrregião do Litoral Paraibano, as quais consomem a polpa endurecida e adocicada dos seus frutos. Além disso, sua casca é utilizada como antiparasítica do couro cabeludo (Braga, 1960; Corrêa, 1974), sendo também empregada na indústria cosmética. Jaracatia spinosa (jaracatiá) apresentou complemento diplóide formado por $2 \mathrm{n}=18$, cromossomos medindo 1,5 a 2,5 $\mu \mathrm{m}$, metacêntricos a submetacêntricos e um par de satélites visíveis (Figura 1E). A presente contagem coincide com a maioria dos registros cromossômicos para o gênero e com a contagem para Caryca dodecaphylla (Fedorov, 1969), uma espécie colocada em sinonímia de J. spinosa (Lorenzi, 1992). A coincidência de números cromossômicos e a proximidade taxonômica entre os gêneros Caryca e Jaracatia tornam possível a utilização das espécies selvagens deste último gênero em programas de melhoramento do mamoeiro (Caryca papaya). O jaracatiá é uma espécie arbórea que produz anualmente uma grande quantidade de frutos comestíveis de polpa adocicada, pouco consumida pela população devido às dificuldades de colheita, por tratar-se de uma árvore alta de tronco espinhoso.

Em Talisia esculenta observou-se $2 \mathrm{n}=32$, confirmando a única contagem para a espécie (Carvalheira et al., 1991) e a estabilidade cromossômica numérica do gênero (Lombello \& Forni-Martins, 1998). O cariótipo é constituído por cromossomos medindo de 1,0 a 2,0 $\mu \mathrm{m}$, sendo os 13 pares maiores metacêntricos e submetacêntricos, e os três pares menores, acrocêntricos, um dos quais, satelitado (Figura 1F). Em outras Sapindaceae, predominam $n=10 ; 11$ e 12, nas espécies trepadeiras, e $n=14 ; 15$ e 16, nas arbustivas e arbóreas, o que aparentemente é um caráter derivado na família (Forni-Martins \& Martins, 2000). A ocorrência de um mesmo número cromossômico em espécies próximas de Talisia indica ser possível a hibridização interespecífica, possibilitando muitas opções de melhoramento genético para a obtenção de linhagens com melhores características comerciais, mais produtivas e resistentes. Os frutos dessa e de outras espécies 
são comestíveis (Silva et al., 1994), comercializados em forma de "cachos" nas feiras livres, estradas e esquinas das cidades das regiões Norte e Nordeste do País, sendo também cultivada em pomares domésticos.

\section{CONCLUSÕES}

1. As espécies selvagens analisadas, em termos estritamente cariológicos, podem ser utilizadas em programas de melhoramento.

2. A ausência de contagens prévias na maioria dos táxons analisados indica a necessidade de análises cromossômicas mais extensas em espécies de frutíferas nativas para que se tenha um quadro mais claro de suas potencialidades de uso em programas de melhoramento.

3. A ocorrência de heteromorfismo em um par cromossômico satelitado de Guazuma ulmifolia parece indicar a ocorrência de uma inversão pericêntrica heterozigota.

\section{REFERÊNCIAS}

ATCHISON, E. Chromosome Numbers in the Myrtaceae. American Journal of Botany, Columbus, v. 34, n. 3, p.159$164,1947$.

BRAGA, R. Plantas do Nordeste: especialmente do Ceará. Coleção Mossoroense, Natal, v.315, p.379-380, 1960.

CARVALHEIRA, G.M.G.; GUERRA, M.; SANTOS, G.A. DOS; ANDRADE, V.C.; FARIAS, M.C.A. DE. Citogenética de angiospermas coletadas em Pernambuco-IV. Acta Botânica Brasílica, Porto Alegre, v.5, n.2, p.37-51, 1991.

CARVALHO, R.; SOARES FILHO, W.S.; BRASILEIRO VIDAS, A.C.; GUERRA, M. The relationships among lemons, limes and citron: a chromossomomal comparison. Cytogenetic and Genome Reseach, Basel, v.109, n.1, p.276-282, 2005.

CORRÊA, M.P. Dicionário das plantas úteis do Brasil. Rio de Janeiro: Imprensa Oficial, 1974. v.5, p.280-281.

ÉDER-SILVA, ERLLENS; BRUNO, R.L.A.; FELIX, L.P.; DORNELAS, C.S.M.; SOUZA, M.A.; RODOLFO JÚNIOR, R.; SOUZA, G.G. Morfologia de fruto e plântula e caracterização fisico-química de frutos de araçá-boi (Psidium arboreum). In: SIMPÓSIO BRASILEIRO PÓS-COLHEITADE FRUTOS TROPICAIS, 1., 2005, João Pessoa. Anais... João Pessoa: Embrapa/UFPB/UFS/SBF, 2005a. 6p.

ÉDER-SILVA, ERLLENS; BRUNO, R.L.A.; FELIX. L.P.; SILVA, S.M.; NOGUEIRA, D.H.; SOUZA, M.A. Caracterização físico-química de frutos de banana-de-raposa (Bromelia karatas 1.). In: SIMPÓSIO BRASILEIRO PÓS-COLHEITADE FRUTOS TROPICAIS, 1., 2005, João Pessoa. Anais... João Pessoa: Embrapa/UFPB/UFS/SBF, 2005b. 6p.

FEDOROV, A.A. Kromosomnye chisla tsvetkovykh rastenii: chromosome numbers of flowering plants. Leningrad: Academy of Sciences USSR, 1969.

FELIX, L.P.; GUERRA, M. Cytogenetic studies on especies of Habenaria (Orchidoideae: Orchidaceae) occurring in the Northeast of Brazil. Lindleyana, West Palm Beach, v.13, n.4, p.224-230, 1998.
FERNANDEZ, A.; KRAPOVICKAS, A. Cromosomas y evolution en Arachis (leguminosae). Bomplandia, Cordoba, v.8, n.1, p.187-220, 1994.

FORMINAYA, A.; LINARES, C.; LOARCE, Y. FERRE, E. Microdissection and microcloning of plant chromossomes. Cytogenetic and Genome Reseach, Basel, v.109, p.8-14, 2005.

FORNI-MARTINS, E.R.; MARTINS, F.R. Chromosome studies on Brazilian cerrado plants. Genetics and Molecular Biology, Ribeirão Preto, v.23, n.4, p.947-955, 2000.

GOLDBLATT, E.P. Index tho plant chromosome numbers 19751978. Monographs in Systematic Botany, St Louis, v.5, n.1, p.78-82, 1981.

GOLDBLATT, E.P. Index tho plant chromosome numbers 19821993. Monographs in systematic Botany, St Louis, v.13, n.1, p.68-78, 1985.

GOLDBLATT, E.P.; JOHNSON, D.E. Index tho plant chromosome numbers 1990-1991. Monographs in Systematic Botany, St Louis, v.51, n.1, p.90-98, 1994.

GUERRA, M. Mitotic and meiotic analysis of a pericentric inversion associated with a tanden duplication in Eleutherine bulbosa. Chromosoma, Berlin, n.97, v.1, p.8087, 1988.

GUERRA, M.; NOGUEIRA, M.T.M. The cytotaxonomy of Emilia (Asteraceae: Senecioneae) species occuring in Brazil. Plant Systematics and Evolution, Vienna, v.170, p.229-236, 2001.

GUERRA, M.; SOUZA, M.J. Como observar cromossomos: um guia de técnicas em citogenética vegetal, animal e humana. Ribeirão Preto: Ed. FUNPEC, 2002. 131p.

LOMBELLO, R. A., FORNI-MARTINS, E. R. Cytological studies in climbers of a brazilian forest reserve. Cytologia, Tokyo, v.63, n.1, p.415-420, 1998.

LORENZI, H. Árvores brasileiras. Nova Odessa: Ed. Plantarum, 1992. p.315-322.

MANICA, I. Frutas nativas, silvestres e exótica 1: técnica de produção e mercado. Porto Alegre: Ed. Cinco Continentes, 2000.327p.

MELO, N.F.; GUERRA, M. Variability of the 5S and 45S rDNA Sites in Passifloraceae L. Species with Distinet Base Chromosome numbers. Annals of Botanical, London, v.92, p.309-316, 2003.

MELO, N.F.; CERVI, A.C.; GUERRA, M. Karyology and citotaxonomy of the genus (Passiflora spp.). (Passifloraceae). Plant Systematics and Evolution, Vienna, v.226, p.69-84, 2001.

MELO, N.F.; GUERRA, M.; BENKO-ISEPPON, A.M.; MENEZES, N.L. Cytogenetics and cytotaxonomy of vellozaceae. Plant Systematics and Evolution, Vienna, v.204, p.257-173, 1997.

PAGGIO, L.; GONZALES, G.; CONFALONIERI, V.; COMAS, C.; NARANJO, C.A. The genome organization and diversification of maize and its allied species revisited: evidences from classical and Fish-Gish cytogenetic analysis. Cytogenetic and Genome Reseach, Basel, v.109, p.259-267, 2005.

PEDROSA, A. Chromosomal organization and physical mapping in legumes. 2002. 143f. Tese (Doutorado) - Universidade de Vienna. Vienna, 2002. 
PEDROSA, A.; SANDAL, N.; STOUGAARD, J.; SCHWEISER, D.; BACHMAIR; A. Chromosomal map of the model Legume Lotus japonicus. Genetics, Atlanta, v.161, p.1661-1672, 2002. PIEROZZI, N.I.; CRUZ, N.D. Número e identificação de cromossomos de Genipa americana L. (Rubiaceae) através de técnica de bandeamento C. Ciência e Cultura, São Paulo,v.40, supl., p.885-888. 1998.

SILVA, J. A.; SILVA, D. B.; JUNQUEIRA, N. T. V.; ANDRADE, L. R. M. Frutas nativas do cerrado. Planaltina: EMBRAPA/ CPAC, 1994. $166 \mathrm{p}$.

STEBBINS, G.L. Cromossomal variation in higher plants. London: Edward Arnold, 1971.216p.

STUESSY, F.O.D.F. Plant taxonomy: a sistematic evolution of comparative data. New York: Columbia University Press, 1990.514p. 